\title{
Ecological propriety and architecture
}

\author{
V. A. Metallinou \\ Hellenic Society for the Protection of the Environment and Cultural \\ Heritage, Thessaloniki Branch, Greece
}

\begin{abstract}
For centuries, building has been seen largely as a way of living apart from the environment and dominating nature. This has turned out to be a pyrrhic supremacy and the current ecological crisis has motivated many professionals and academics to re-evaluate the fundamental premises of how buildings are designed and produced.

The potential of buildings to cause global environmental damage was first acknowledged during the 1980s and out of this realization, the concept of "sustainability" emerged which now enjoys a central place in the discourse.

There are clear connections between the effects of globalization and architectural practice. There is a direct link between the destruction of the rainforests and how we build and with which materials; and the erosion of the ozone layer has led to a reassessment of how energy is used in buildings.

The issues that bioclimatic buildings and settings address are essentially threefold: energy, health and wellbeing and sustainability.

Yet, eco architecture is not only a matter of specific design choices that lead (most of the time) to specific high tech building products, but the appropriate mentality that emancipates a specific attitude of dealing with building within nature.

In doing so, regional and national planning should surely lay emphasis on maximizing ambient energy and at the local level, planning should strive to increase density in urban areas to combat the increasing suburbanization, as a means to protect surrounding nature - the raison d' etre of the city.

To Vitruvious' Firmitas, Utilita and Venustas (strength, functionality and beauty) a further criterion has to be added - restitutes (restitution) in which the act of building enhances the environment in an ecologically responsible manner. Keywords: globalization and architectural practice, building and energy, building within nature, eco-architecture, energy, health and wellbeing and sustainability.
\end{abstract}




\section{Introduction}

For centuries, building has been seen largely as a way of living apart from the environment and dominating nature. This has turned out to be a pyrrhic supremacy and the current ecological crisis has motivated many professionals and academics to re-evaluate the fundamental premises of how buildings are designed and produced. Underscoring technical efforts to reconstitute the built environment is the elusive but critically important concept of sustainable development.

Across a wide range of disciplines including architecture, a new attitude, conjecturing a built environment that mimics and complements rather than conflicts with nature, is emerging, as a vital goal of current theory and practice.

Notions of sustainability are not a preoccupation of recent history. The medieval monasteries of Europe and in the Balkans, led their unique ecological paradigm centuries ago. They produced their own food, created buildings from local materials, captured and recycled water and developed renewable energy technologies such as water and windmills. These highly structured societies, took care of the sick and elderly, cultivated land according to ecological principles and had a humane approach to animal husbandry.

Such practices still obtain in rural communities in Latin America, Africa and Asia and often appropriate selectively aspects of contemporary technology.

For instance, in the desert regions of North Africa, doctors still travel by camel to visit remote villages. It's a tableau unchanged for generations, but nowadays the camel is equipped with a GPS antenna on its head to signal its geographical position, and a photovoltaic panel on its back that provides energy to run a fridge containing medicines to treat the sick.

This instinctive, almost poetic synthesis of tradition and technology could have wider implications for the sclerotic First World, as "sustainable development" is not a term one hears in such settlements, but in reality, these are places from which the rest of humanity could usefully draw lessons rather than seeking to "improve" them. There is a danger of a nostalgic glorification of some isolated, eco - responsive aboriginal society, totally removed from our debauched, technologically sophisticated milieu. On the other hand, the lessons of these civilizations and their cosmology reveal a wealth of insights into the evolution of the human habitat that cannot be ignored. They provide instructive examples of how to deal with climate and demonstrate ideas, attitudes and lowtech solutions that can be usefully incorporated into contemporary shelter. Equally importantly, these cultures offer the basis for rethinking humankind's relationship with the planet.

\section{The line of history}

In the Pioneers of Modern Design, Nikolaus Pevsner identified the Arts and Crafts Movement flourish in Britain in the decades either side of 1900s, as one of the major influences upon the emergence of the Modern Movement. 
In the ideas of William Morris and their translation into architectural form and language is possible to recognize antecedents of Modernist Architectural theory and practice. Morris' theories established the principles of fitness for purpose and the integrity of materials as a fundamental importance in the design of all articles of use. To these, architects of the Arts and Crafts Movement added a sense of response to place in applying Morris' ideas to the design of buildings. In their specific interpretation and application of principle, they placed particular emphasis upon the question of orientation in determining the placement of buildings and particularly houses upon their sites and the position of the principal rooms. More over, with the use of the bay window, they concentrated the main areas of glazing towards the sun.

The environmental credentials of this architecture extend beyond observance of orientation, into a complex and subtle setting for domestic inhabitation. Spaces may be closed-off or opened-up in response to environmental and social requirements and the garden is consistently conceived as an essential complement to the interior spaces and not as an added-on afterthought.

In more modern terminology, what we find here is an environment which is climate-responsive, spatially and temporally diverse, user-controlled, adaptable and sustainable. We also perceive a conception of nature of architecture that predates the reductive processes of $20^{\text {th }}$ century, in which questions of environment are comprehended within an all-embracing synthesis of the cultural, social, technological and aesthetic.

By the late 1920s, the events traced by Pevsner in the Pioneers, had led to the transformation of the language of architecture from a traditionally grounded synthesis to a new analytical clarity - the Modern Movement.

The methods of architectural science in the application of quantified standards in the functional needs of buildings and their users attract particular interest in the relationship between design and the environmental principles, where quantification is placed in the context of a particular vision of the wholeness of architectural enterprise.

As the idea of research mainly took roots in schools of architecture, so environmental studies started gaining importance among the academia as well as in policy making bodies. The continuity of the field in which we all work, is expressed clearly in the discipline of the philosophy of science of the era. Thinkers such as Karl Popper and Thomas Kuhn describe the way in which science proceeds systematically within the discipline of prevailing paradigms, using rigorous procedures and accumulating a body of knowledge, which serves the solution of new emerging problems. The hypothesis of research programmes, introduced by Imre Lakatos was used as the core theory for proposing in the 1980s the Architectural Research Programmes as the way to understand the development of our discipline-Stanford Anderson - Vivianna Metallinou Libero Andreotti, MIT, 1982. The whole enterprise made possible to bring into focus all of the variables and processes which occur in the environmental system of a building along with the central role of the occupant and the contextual studies of the special relation between site and building, prepared the way of 
architecture to take advantage of theory and practice in the environmental research (Regionalism and so).

\section{Sustainability and architecture}

With the energy crisis of the 1970s, the issue brought into focus was the relationship of environmental design to the energy demand of building. From this, a new parameter entered the equation of architecture: the concern to explore the relationship between the form and materiality of buildings and their energy consumption. Physical, mathematical and computer modeling of building environment in the architecture schools, proposed either quite simple graphical representations or experimental structures for use by designers.

Terms such as "low-energy architecture", "passive solar design" and "bioclimatic architecture", entered the lexicon and importantly, redefined the agenda of both research and practice.

The potential of buildings to cause global environmental damage was first acknowledged during the 1980s and out of this realization, emerged the concept of "sustainability" which now enjoys a central place in the discourse. There are clear connections between the effects of globalization and architectural practice. There is a direct link between the destruction of the rainforests and how we build and with which materials; and the erosion of the ozone layer has led to a reassessment of how energy is used in buildings.

Architects, however, are dependent on other bodies for the gathering and assessment of data, for the analysis of causes of degradation and for proposals for its reversal. These bodies come in various forms: ${ }^{L}$ international agencies (the Club of Rome) national ministries, research institutes, non-government organizations NGO, independent consultancies and individuals. It is left to the architects to make sense of it all, in the context of a building where energy and environment may be seen as important issues, but where there are other equally important requirements (from the client) and where at the end of the day the building must be a complete architectural statement.

It has been said that architecture is the mother of the arts, thereby claiming for it both maternal and aesthetic ascendancy. If this is so, the matriarch in architecture holds in balance the well-being of another mother figure: MotherEarth, while the art in architecture, uniquely has to meet the complex demands of use as well as transporting the senses. It is on the relationship of these two roles architecture as an art of function and architecture as environmental custodian, that the whole discussion is focused.

Bioclimatic architecture is seen as a reaction to the predicament of environmental depredation in the same way organic farming is a reaction to the dominance of intensive, chemically based agriculture. And worthy but dull enough it is positioned at the fringe of the main production. The attitude to attain yet the form of another movement leads it to another categorization that separates and marginalizes it again. 


\section{The proposal}

... "In doing a memorial I started with a room and a garden. That was all I had. Why did I choose a room and a garden as a point of departure? Because the garden is a personal gathering of nature and the room is the beginning of architecture" starts Luis Kahn in his "Between Silence and Light".

The dialogue between architecture and nature is as old as architecture itself. It is only in recent history that this happy interaction has been extinguished. Until then, both aesthetic and functional attributes of architecture were inextricably linked to nature. Seen as such, bioclimatic architecture represents an overdue return to and a broadening of the innate and continuing exchange. It lies squarely in the mainstream of architecture and is neither expedient reparation nor arcane cult. Architecture is depended on a satisfactory reconciliation of the intuitive with the rational. A building has to be both a poem and a machine. Yet, few buildings achieve such a status. There are those that are sensually stimulating but lack sound construction and those that answer successfully practical needs but fail to generate an emotional charge.

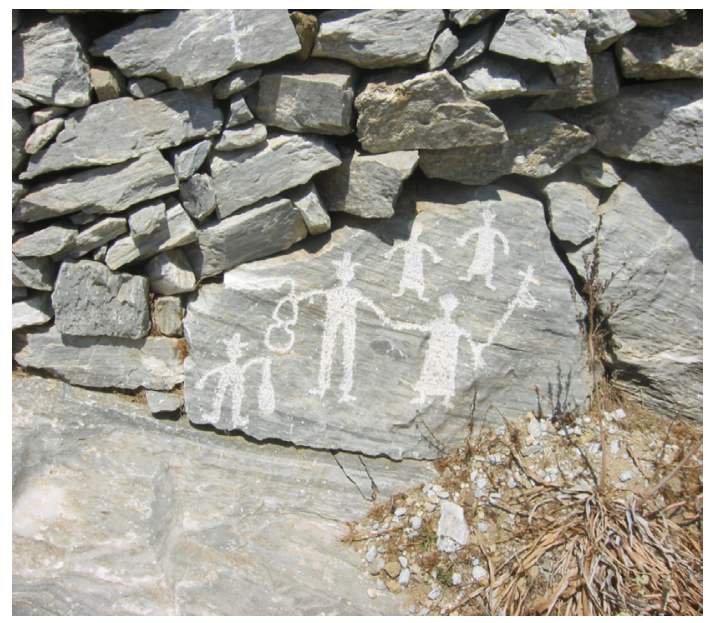

Figure 1.

Axiomatic to arriving at an inspirational balance between sense and sensibility are two relationships: that of building to site and both of these to nature itself. The capture of the genius loci is the content that makes a chosen site special and distinctive. The relationship of building and site to the wider natural environment is again subject to the intuitive and the rational. The art of architecture therefore, has to embrace both the inspirational and the analytical and architectural response to our environmental predicament will also reflect this dichotomy. 


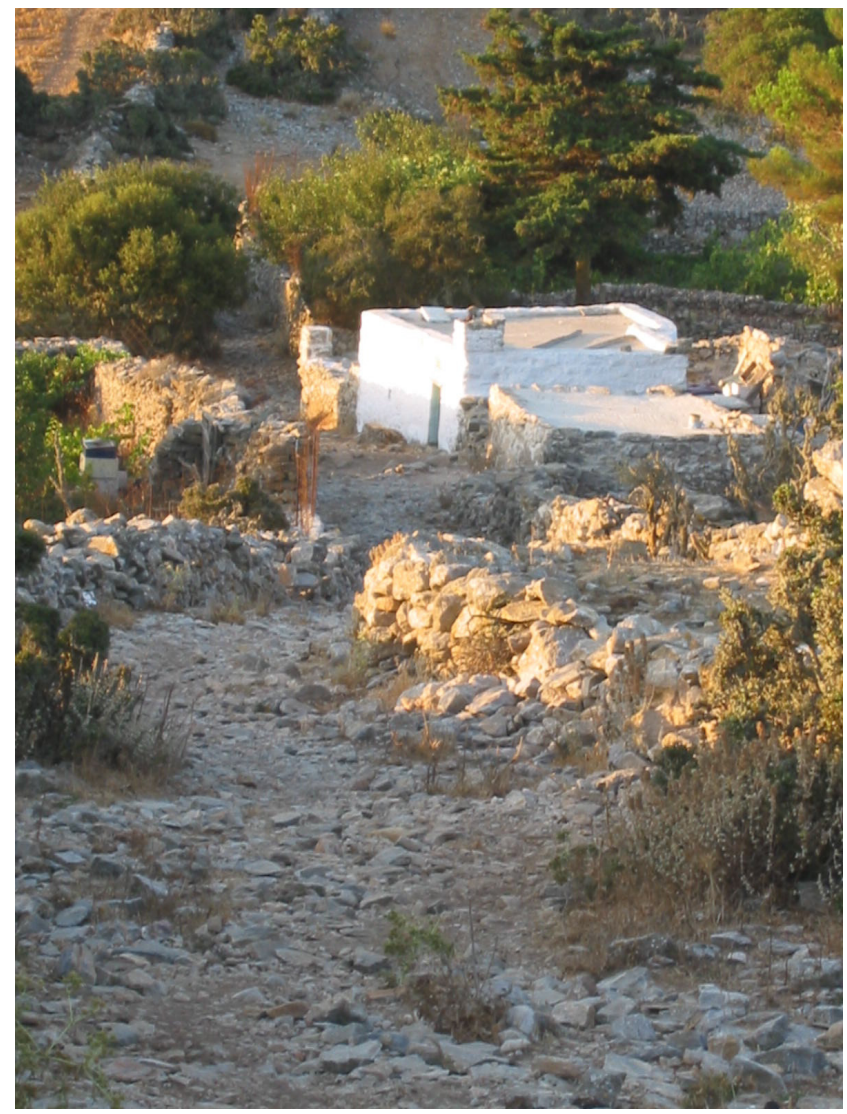

Figure 2.

..."First we had nature. And then came the Environment. Environment is the smoke, humanity has put on nature: the people who used Latin had no word for environment- they only knew natura" [1].

It is more than obvious that we have to agree on what we mean by the newly defined terms of our discussion. Both environment and sustainability mean numerous and different things. What we refer to as bioclimatic is an approach to design that is inspired by nature and which applies a sustained logic to every aspect of the project, focused on minimizing environmental depredation and encouraging a sense of well-being.

The issues that bioclimatic buildings and settings address are essentially threefold: energy, health and wellbeing and sustainability. A key aspect in designing a bioclimatic building is determining its comfort criteria, as perception of comfort varies considerably from individual to individual and between ethnic groups who become more habituated to local climatic conditions. It also varies between countries and continents according to the level of development each of them is having. 
The extraction of potent architectural expression from underlying social theory and technical innovation is the stuff of architecture and as noted, a characteristic of mature bioclimatic architecture.

\section{New partners on design}

Architecture as a discipline to itself is only one facet of the issue. In the 2002 UIA World Congress in Berlin, the main theme elaborated by the organizers was that architecture is subjected to take into direct consideration the growing importance of new technology in building methods as well as the new materials available in the market. As a result of that, even from the time of the idea elaboration, architecture is the outcome of a team - work between architect and the building contractor (or company) and the scheme has come over to embrace the whole design process. We still enjoy architecture in the Bazaars of Architecture - the cities as the theme of the last UIA 2005 World Congress in Istanbul presented, with the unrivaled star system architecture to claim one aspect of the production and the Euphoria Manifestoes on the other hand to return the discussion on the sustainable aspect (Richard Senett).

The growing sensitivity and public interest, to the appreciation of cultural heritage that is being extended to include and embrace all interesting manmade structures since the last decade, is shaping another strong aspect on the issue of sustainability on architecture. Networking in the field of heritage is a practice that strengthens attitudes towards the appreciation and use of culture as a driving force for shaping the present. Fighting for the preservation of the cultural heritage of one's millieu, helps shaping the appropriate mentality for an eco attitude at first, which will be followed by eco-architecture, eco-mechanics, ecoagriculture perhaps.

Sustainability considered from this point of view, functions as a means of cultural consistency. Design gains a meaning as a chain of cultural ${ }^{\mathrm{L}}$ continuity and linked to the social development policy. Within these qualities, the sustainability can be interpreted:

- As a means of integration between society and the profession

- As a means of surviving the cultural values and tradition

- To make the user an effective part of the design process

- To improve the communication among different disciplines and scientific fields

The important point is to bring together the civilian contribution with the idea of ecology and to initiate the participation and support processes on the issue.

When developed with a method that can be used as a platform to raise the voice of the free thought, this initiative will become a contribution for the culture of democracy and will propose a reform process in the field of interaction. The sustainability approach is before all a reform process in thought. Like any other processes it necessitates a political choice or stance. The most significant determiner is the logic of the process and therefore it is inevitable for the economic and political powers to exist in this transformation and reproduction process determinedly and take the necessary responsibility. 
Against the multidimensional erosion emerged with globalization and spread to several fields, the attitude of the architect and designer gradually gains significance and their political choices can become a determining factor for the process.

Eco architecture is not only a matter of specific design choices that lead (most of the times) to specific high tech building products, but the appropriate mentality that emancipates a specific attitude of dealing with building within nature.

Coming from a country known for the specific quality of place, I believe that eco architecture has above all to submit seriously to the attitude of the protection of everything that lays there, in order to keep trace of a development that need not research booths to come up with the proper and right. In doing so regional and national planning should surely lay emphasis on maximizing ambient energy while at local level, planning should strive to increase density in urban areas to combat the increasing suburbanization. Architects must provide geometries of living that offer density with the advantages of suburban life.

A thorough understanding of the principles of passive and active energy conservation should be expected and we should add to Vitruvious Firmitas, Utilita and Venustas (strength, functionality and beauty) a further criterion for judgement of architecture - restitutes (restitution), in which the act of building enhances the environment in ecologically responsible manner.

The line I am drawing here is a paradigm from the holistic traditional procedures, indicating the uninterrupted continuity of the field in which we work and demonstrating the prevailing belief that the problem can be dealt on. By exploring notions of appropriate rather than high technology and create buildings that are not only harmoniously integrated with the landscape but also climatically responsive, embodying the soul and substance of an emerging ecological propriety that gives great hope for the future in a humane, inventive, radical and passionate architecture that acknowledges the world beyond the glossy magazine pages. We, architects, cannot transform society, but we can certainly transform architecture. And by doing so, we will change other forms of production too.

\section{References:}

[1] Metzger Gustav, Symposium on Sustainability, The Architectural Association, London, UK. 1996.

[2] Dr. Incedayi Deniz, Sustainability as a form of conduct in the context of environmental sensibility, proc. of the $1^{\text {st }}$ international conf. of architects "Architecture and sustainable development on the Balkans", Sofia, pp 19, 2004.

[3] Dr. Slaev Alexander, Balkan architecture and architects - working towards sustainable development of southeastern Europe, proc. of the $1^{\text {st }}$ international conf. of architects "architecture and sustainable development on the Balkans”, Sofia, pp 19-26, 2004.

[4] Davey Peter, Designing our future, The Architectural Review124, pp 2627, January 2001. 